\title{
Einstein-Maxwell Fields of Embedding Class One
}

\author{
C. D. Collinson \\ Department of Applied Mathematics, \\ The University of Hull
}

Received December 1, 1967

\begin{abstract}
It is shown that a necessary condition for an Einstein-Maxwell field to be of embedding class one is that the electromagnetic field and Weyl tensor are both null (and non-zero). All Einstein-Maxwell fields of embedding class one are, in principle, obtained.
\end{abstract}

\section{Introduction}

The investigation carried out in this paper was motivated by the desire to determine whether the dimensionality of embeddings, discussed elsewhere [1], of certain Einstein-Maxwell fields is minimal. Necessary and sufficient conditions for embedding class one have been found by Thomas [2]. However, these conditions involve extremely heavy algebraic manipulations and have not been much used by other workers. In fact, several papers have appeared recently discussing space-times of embedding class one, for instance Szekeres [3] and StePHANI [4]. In particular Stephani has investigated null electromagnetic fields of embedding class one. The present paper completes these investigations and generalizes them to the non-null case. The results can, in part, be stated as the

Theorem. Solutions of the Einstein-Maxwell field equations can be embedded (locally and isometrically) in a five dimensional pseudo-euclidean space only if the electromagnetic field and the Weyl tensor are both null and non-zero.

The null tetrad notation of Newman and Penrose [5] is used to prove this theorem. The notation is based on a tetrad of vectors $l^{\alpha}, n^{\alpha}, m^{\alpha}, \bar{m}^{\alpha}$ satisfying the orthonormality conditions

$$
l^{\alpha} n_{\alpha}=-m^{\alpha} \bar{m}_{\alpha}=1,
$$

all other contractions being zero. Throughout this paper Greek letters $\alpha, \beta, \gamma, \ldots$ denote tensor indices (and range from 1 to 4 ), Roman letters $m, n, p, \ldots$ denote tetrad indices (and range from 1 to 4 ) whilst capital letters $Q, R, S, \ldots$ denote tensor indices in the embedding space (and range from 1 to 5). The tetrad components of the Weyl tensor can be

1 Commun. math. Phys., Vol. 8 
written in terms of the five complex scalars $\psi_{0}, \psi_{1}, \psi_{2}, \psi_{3}, \psi_{4}$ whilst the tetrad components of the Ricci tensor can be written in terms of the ten scalars $\phi_{m n}, \Lambda$ (here alone the indices $m, n$ take the values $0,1,2$ ). Solutions of the Einstein-Maxwell field equations are characterized by

$$
\Lambda=0, \quad \phi_{m n}=\phi_{m} \bar{\phi}_{n}
$$

where $\phi_{m}$ are the following tetrad components of the electromagnetic field tensor $F_{\alpha \beta}$,

$$
\phi_{0}=F_{\alpha \beta} l^{\alpha} m^{\beta}, \phi_{1}=1 / 2 F_{\alpha \beta}\left(l^{\alpha} n^{\beta}+\bar{m}^{\alpha} m^{\beta}\right) \quad \text { and } \quad \phi_{2}=F_{\alpha \beta} \bar{m}^{\alpha} n^{\beta} .
$$

The elegance of the Newman-Penrose notation is in the introduction of ten functions, called the spin coefficients, which are defined in terms of the complex Ricci rotation coefficients $\gamma_{m n p}$ by

$$
\begin{aligned}
& \varkappa=\gamma_{131}, \quad \pi=-\gamma_{241}, \quad \varepsilon=1 / 2\left(\gamma_{121}-\gamma_{341}\right), \quad \varrho=\gamma_{134}, \quad \lambda=-\gamma_{244}, \\
& \sigma=\gamma_{133}, \quad \mu=-\gamma_{243}, \quad \alpha=1 / 2\left(\gamma_{124}-\gamma_{344}\right), \quad \beta=1 / 2\left(\gamma_{123}-\gamma_{343}\right), \\
& \nu=-\gamma_{242}, \gamma=1 / 2\left(\gamma_{122}-\gamma_{342}\right) \text { and } \tau=\gamma_{132} .
\end{aligned}
$$

An explicit notation is sometimes used for intrinsic derivatives, namely

$$
D \phi \equiv \phi,{ }_{i} l^{i}, \Delta \phi \equiv \phi,{ }_{i} n^{i} \text { and } \delta \phi \equiv \phi,{ }_{i} m^{i} .
$$

Continual use will be made of the Newman-Penrose field equations, the commutation relations for intrinsic derivatives, the EinsteinMaxwell field equations and, finally, the Bianchi identity in the presence of an electromagnetic field. All these equations are to be found in the references [5] and [6] and will not be reproduced here.

\section{The Gauss-Codazzi Equations}

It is well known [7] that a space-time is of embedding class one if and only if there exists a symmetric tensor $a_{m n}$ satisfying the following equations.

Gauss equation:

Codazzi equation:

$$
R_{m n p q}=2 e a_{m[p} a_{q] n} .
$$

$$
a_{m[n ; p]}-\gamma_{[n p]}^{q} a_{m \alpha}+\gamma_{m[n}^{q} a_{p] q}=0 .
$$

In the above $R_{m n p q}$ is the curvature tensor of the space-time, $e= \pm 1$ and square brackets denote antisymmetrization. These equations are the integrability conditions of the differential equations

and

$$
y_{; m n}^{Q}-y_{; p}^{Q} \gamma_{m n}^{p}=e a_{m n} \eta^{Q}
$$

$$
\eta_{; m}^{Q}=-a_{p m} y_{;}^{Q p} \text {. }
$$

Here $\eta^{Q}$ is a vector normal to the space-time and $y^{Q}$ are coordinates in the embedding space. 
A necessary condition for embedding class one can be obtained in the following straight forward manner. Define

$$
T_{m n p q}=\varepsilon^{s t u v} R_{s t m p} R_{u v n q} .
$$

If the Gauss equation is satisfied

Therefore

$$
T_{m n p q}=4 \varepsilon^{s t u v} a_{s m} a_{t p} a_{u n} a_{v q}=4|a| \varepsilon_{m n p q} .
$$

$$
T_{m n p q}+T_{m n q p}=0 .
$$

The condition (2.6) is very useful when solving the equations (2.1). Contracting (2.6) on $p$ and $q$ gives

$$
\varepsilon^{s t u v} R_{s t m}^{q} R_{u v n q}=0 .
$$

This can be written in terms of the Weyl tensor $C_{m n p q}$ and Ricci tensor $R_{m n}$ as

$$
C^{u^{*} v p q} C_{u v p q}=R_{p}^{t} C_{n t}^{p^{*} m}=0,
$$

where * denotes the dual tensor. The components of the Gauss equation (2.1) can be written explicitly as

$$
\begin{aligned}
& e\left(a_{13} a_{13}-a_{11} a_{33}\right)=\psi_{0} \\
& e\left(a_{12} a_{13}-a_{11} a_{23}\right)=\psi_{1}+\phi_{01} \\
& e\left(a_{12} a_{34}-a_{14} a_{23}\right)=\psi_{2}+2 \Lambda \\
& e\left(a_{12} a_{24}-a_{22} a_{14}\right)=\psi_{3}+\phi_{21} \\
& e\left(a_{24} a_{24}-a_{22} a_{44}\right)=\psi_{4} \\
& e\left(a_{13} a_{34}-a_{14} a_{33}\right)=\psi_{1}-\phi_{01} \\
& e\left(a_{11} a_{34}-a_{13} a_{14}\right)=-\phi_{00} \\
& e\left(a_{13} a_{23}-a_{12} a_{33}\right)=-\phi_{02} \\
& e\left(a_{22} a_{34}-a_{23} a_{24}\right)=-\phi_{22} \\
& e\left(a_{24} a_{34}-a_{23} a_{44}\right)=\psi_{3}-\phi_{21} \\
& e\left(a_{11} a_{22}-a_{12} a_{12}\right)=-\psi_{2}-\bar{\psi}_{2}-2 \phi_{11}+2 \Lambda \\
& e\left(a_{33} a_{44}-a_{34} a_{34}\right)=-\psi_{2}-\bar{\psi}_{2}+2 \phi_{11}+2 \Lambda
\end{aligned}
$$

while the components of equation (2.6) can be written

$$
\begin{gathered}
\phi_{02}\left(\psi_{2}-\bar{\psi}_{2}\right)-2 \psi_{1} \phi_{12}+2 \bar{\psi}_{3} \phi_{01}-\bar{\psi}_{4} \phi_{00}+\psi_{0} \phi_{22}=0 \\
-\phi_{00} \phi_{12}-\phi_{02} \phi_{10}+\psi_{1}\left(-\psi_{2}+4 \Lambda\right)+2 \phi_{01} \phi_{11}+\psi_{0} \psi_{3}=0 \\
-\phi_{22} \phi_{10}-\phi_{20} \phi_{12}+\psi_{3}\left(-\psi_{2}+4 \Lambda\right)+2 \phi_{21} \phi_{11}+\psi_{4} \psi_{1}=0 \\
\phi_{01}\left(\psi_{2}+2 \bar{\psi}_{2}\right)-\bar{\psi}_{3} \phi_{00}-\bar{\psi}_{1} \phi_{02}-2 \psi_{1} \phi_{11}+\psi_{0} \phi_{21}=0 \\
\phi_{21}\left(\psi_{2}+2 \bar{\psi}_{2}\right)-\bar{\psi}_{2} \phi_{22}-\bar{\psi}_{3} \phi_{20}-2 \psi_{3} \phi_{11}+\psi_{4} \phi_{01}=0
\end{gathered}
$$




$$
\begin{gathered}
-\phi_{00}\left(\psi_{2}-\bar{\psi}_{2}\right)+2 \psi_{1} \phi_{10}-2 \bar{\psi}_{1} \phi_{01}+\bar{\psi}_{0} \phi_{02}-\psi_{0} \phi_{20}=0 \\
-\phi_{22}\left(\psi_{2}-\bar{\psi}_{2}\right)+2 \psi_{3} \phi_{12}-2 \bar{\psi}_{3} \phi_{21}+\bar{\psi}_{4} \phi_{20}-\psi_{4} \phi_{02}=0 \\
\psi_{0}\left(\psi_{2}+2 \Lambda\right)-{\psi_{1}}^{2}+\phi_{01} \phi_{01}-\phi_{00} \phi_{02}=0 \\
\psi_{4}\left(\psi_{2}+2 \Lambda\right)-{\psi_{3}}^{2}+\phi_{21} \phi_{21}-\phi_{22} \phi_{20}=0 \\
\left(\psi_{2}+\bar{\psi}_{2}+2 \phi_{11}-2 \Lambda\right)\left(\psi_{2}-\bar{\psi}_{2}\right)-\left(\psi_{1}+\phi_{01}\right)\left(\psi_{3}+\phi_{21}\right) \\
\quad+\left(\bar{\psi}_{1}+\phi_{10}\right)\left(\bar{\psi}_{3}+\phi_{12}\right)=0 \\
\left(\psi_{2}+\bar{\psi}_{2}-2 \phi_{11}-2 \Lambda\right)\left(\psi_{2}-\bar{\psi}_{2}\right)-\left(\psi_{1}-\phi_{01}\right)\left(\psi_{3}-\phi_{21}\right) \\
\quad+\left(\bar{\psi}_{1}-\phi_{10}\right)\left(\bar{\psi}_{3}-\phi_{12}\right)=0 \\
\left(\psi_{2}+\bar{\psi}_{2}+2 \phi_{11}-2 \Lambda\right)\left(\psi_{2}+\bar{\psi}_{2}-2 \phi_{11}-2 \Lambda\right)-\psi_{4} \psi_{0} \\
\quad+\phi_{00} \phi_{22}-\left(\bar{\psi}_{1}+\phi_{10}\right)\left(\bar{\psi}_{3}-\phi_{12}\right)+\phi_{02} \phi_{20} \\
\quad+\left(\bar{\psi}_{2}+2 \Lambda\right)^{2}-2\left(\psi_{2}+2 \Lambda\right)\left(\bar{\psi}_{2}+2 \Lambda\right) \\
-\left(\bar{\psi}_{3}+\phi_{12}\right)\left(\bar{\psi}_{1}-\phi_{10}\right)=0 .
\end{gathered}
$$

\section{Algebraically General Electromagnetic Fields}

If a solution of the Einstein-Maxwell field equations represents an algebraically general electromagnetic field then there exists two null vectors satisfying the equation

$$
k^{\alpha} F_{\alpha[\beta} k_{\gamma]}=0 \text {. }
$$

Choosing these vectors as the tetrad vectors $l^{\alpha}$ and $n^{\alpha}$ gives

$$
\phi_{0}=\phi_{2}=0 \text {. }
$$

Without loss of generality it can be assumed that $\phi_{1}$ is non-zero. Eqs. (2.19), ..., (2.30) together with the conditions (1.1) and (3.1) yield either

or

$$
\psi_{2} \neq 0, \quad \psi_{0}=\psi_{1}=\psi_{3}=\psi_{4}=0, \quad 4 \phi_{11}^{2}=3 \psi_{2}^{2}
$$

$$
\psi_{2}=0, \quad \psi_{1}=\psi_{3}=0, \quad 4 \phi_{11}^{2}=-\psi_{0} \psi_{4} .
$$

Substituting (3.2) into the Einstein-Maxwell field equations and into the Bianchi identities gives

$$
\nu=\sigma=\lambda=\varkappa=\mu=\tau=\pi=\varrho=0 .
$$

The Newman-Penrose field equation

$D \mu-\delta \pi=(\bar{\varrho} \mu+\sigma \lambda)+\pi \bar{\pi}-(\varepsilon+\bar{\varepsilon}) \mu-\pi(\bar{\alpha}-\beta)-\nu \varkappa+\psi_{2}+2 \Lambda$

then yields $\psi_{2}=0$ which contradicts (3.2). The case corresponding to (3.3) is far more difficult to deal with. Substituting (3.3) into the Einstein- 
Maxwell field equations gives

$$
\begin{aligned}
D \phi_{1} & =2 \varrho \phi_{1} \\
\Delta \phi_{1} & =-2 \mu \phi_{1} \\
\delta \phi_{1} & =2 \tau \phi_{1} \\
\delta \phi_{1} & =-2 \pi \phi_{1} .
\end{aligned}
$$

Substituting (3.3) into the Bianchi identities gives

with

$$
\begin{aligned}
& D \psi_{0}=(4 \varepsilon+2 \varrho+4 \bar{\varrho}) \psi_{0} \\
& \Delta \psi_{0}=(4 \gamma-2 \mu) \psi_{0} \\
& \delta \psi_{0}=(-4 \bar{\pi}+2 \tau+4 \beta) \psi_{0} \\
& \bar{\delta} \psi_{0}=(4 \alpha-2 \bar{\pi}) \psi_{0}
\end{aligned}
$$

$$
\begin{aligned}
2 \sigma \phi_{1} \bar{\phi}_{1} & =-\mu \psi_{0} \\
2 \varkappa \phi_{1} \bar{\phi}_{1} & =\pi \psi_{0} \\
\nu \psi_{0} & =-2 \tau \phi_{1} \bar{\phi}_{1} \\
\lambda \psi_{0} & =2 \varrho \phi_{1} \bar{\phi}_{1} .
\end{aligned}
$$

Applying the commutators to $\phi_{1}$ and $\psi_{0}$ yields, after simplification,

$$
\begin{aligned}
\Delta \varrho & =\varrho(\gamma+\bar{\gamma})+\pi(\tau+\bar{\tau})+\bar{\tau} \bar{\tau}-\bar{\nu} \bar{\varkappa}+\phi_{11} \\
D \mu & =-\mu(\varepsilon+\bar{\varepsilon})-\tau(\pi+\bar{\tau})-\bar{\tau} \bar{\tau}+\bar{\nu} \bar{\varkappa}-\phi_{11} \\
\delta \varrho & =\varrho(\bar{\alpha}+\beta-\bar{\pi})+\pi \sigma \\
\bar{\delta} \mu & =-\mu(\bar{\beta}+\alpha-\bar{\tau})-\tau \lambda \\
\Delta \pi & =-\nu \varrho-\pi(\bar{\mu}-\bar{\gamma}+\gamma) \\
D \tau & =\varkappa \mu+\tau(\bar{\varepsilon}-\bar{\varepsilon}+\varepsilon) \\
\delta \pi & =-\varrho(\mu-\bar{\mu})-\pi(\beta-\bar{\alpha})-\bar{\mu} \bar{\varrho}+\bar{\lambda} \bar{\sigma}-\phi_{11} \\
\bar{\delta} \tau & =\mu(\varrho-\bar{\varrho})+\tau(\alpha-\bar{\beta})+\bar{\varrho} \bar{\mu}-\bar{\lambda} \bar{\sigma}+\phi_{11} \\
0 & =\pi \bar{\mu}-\bar{\tau} \mu-2 \pi \mu \\
0 & =\tau \bar{\varrho}-\bar{\pi} \varrho-2 \tau \varrho .
\end{aligned}
$$

The Newman-Penrose field equations

$$
\Delta \lambda-\bar{\delta} \nu=-(\mu+\bar{\mu}) \lambda-(3 \gamma-\bar{\gamma}) \lambda+(3 \alpha+\bar{\beta}+\pi-\bar{\tau}) \nu-\psi_{4}
$$

and

$$
D \mu-\delta \pi=(\bar{\varrho} \mu+\sigma \lambda)+\pi \bar{\pi}-(\varepsilon+\bar{\varepsilon}) \mu-\pi(\bar{\alpha}-\beta)+\nu \varkappa+\psi_{2}+2 \Lambda
$$
now give, using (3.5), ..., (3.16),

and

$$
\bar{\varrho} \mu+\varrho \bar{\mu}-2 \varrho \mu-2 \bar{\varrho} \bar{\mu}-\pi \bar{\pi}-\tau \bar{\tau}-2 \tau \pi-2 \bar{\tau} \bar{\pi}=0
$$

$$
\bar{\varrho} \mu+\varrho \bar{\mu}-2 \varrho \mu-2 \bar{\varrho} \bar{\mu}+\pi \bar{\pi}+\tau \bar{\tau}+2 \tau \pi+2 \bar{\tau} \bar{\tau}=0 .
$$


Suppose $\varrho=0$. From Eq. (3.11), $\pi \sigma=0$. If $\pi=0$ Eqs. (3.15) and (3.8) yields $\phi_{11}=0$ which contradicts the hypothesis that $\phi_{1} \neq 0$. If $\pi \neq 0$ then $\sigma=0$ and from Eq. (3.5), $\mu=0$. Eq. (3.9) and (3.10) become, using (3.6) and (3.7),

$$
\begin{aligned}
\pi \tau+\bar{\pi} \pi+\bar{\pi} \bar{\tau}+\bar{\pi} \bar{\tau}+\phi_{11} & =0 \\
-\pi \tau-\bar{\tau} \tau-\bar{\pi} \bar{\tau}-\bar{\pi} \bar{\tau}-\phi_{11} & =0 .
\end{aligned}
$$

The imaginary part of Eq. (3.21) gives

$$
\bar{\pi} \bar{\tau}=\pi \tau,
$$

while Eq. (3.21) plus (3.22) gives

$$
\bar{\pi} \pi=\bar{\tau} \tau .
$$

Substituting these into Eq. (3.19) gives $\tau=-1 / 2 \pi$ and then Eq. (3.24) yields $\pi \tilde{\pi}=0$. This contradicts the hypothesis that $\pi \neq 0$.

Now suppose $\varrho \neq 0$. If $\tau \neq 0$ Eqs. (3.17) and (3.18) yield

$$
3 \bar{\varrho} \mu+\varrho \bar{\mu}-2 \varrho \mu-2 \bar{\varrho} \bar{\mu}=0 \text {. }
$$

Eq. (3.19) plus (3.20) gives

$$
\bar{\varrho} \mu+\varrho \bar{\mu}-2 \varrho \mu-2 \bar{\varrho} \bar{\mu}=0
$$

and comparing these equations, $\mu=0$. Eqs. (3.12) and (3.8) then give $\varrho \tau=0$ which contradicts the hypotheses. If $\tau=0$ then from Eq. (3.18), $\pi=0$. Eqs. (3.15) and (3.16) now become

$$
\begin{aligned}
-\varrho \mu+\varrho \bar{\mu}-\bar{\varrho} \bar{\mu}-\bar{\varrho} \bar{\mu}-\phi_{11} & =0 \\
\varrho \mu-\bar{\varrho} \mu+\bar{\varrho} \bar{\mu}+\bar{\varrho} \bar{\mu}+\phi_{11} & =0 .
\end{aligned}
$$

Eq. (3.27) plus (3.28) gives

$$
\varrho \bar{\mu}=\bar{\varrho} \mu
$$

while the imaginary part of (3.27) gives

$$
\varrho \bar{\mu}=\varrho \mu \text {. }
$$

Substituting these into the sum of the Eq. (3.19) and (3.20) yields

$$
\varrho \bar{\mu}=2 \varrho \mu \text {, }
$$

whence $\mu=0$. From Eq. (3.10) $\phi_{11}=0$ which contradicts the hypothesis that $\phi_{1} \neq 0$. The space-times considered in this section are therefore not of embedding class one.

\section{Null Electromagnetic Fields}

If a solution of the Einstein-Maxwell field equations represents a null electromagnetic field then there exists a null vector satisfying the equations

$$
F_{\alpha \beta} k^{\beta}=F_{[\alpha \beta} k_{\gamma]}=0 .
$$


Choosing this vector as the tetrad vector $l^{\alpha}$ gives

$$
\phi_{0}=\phi_{1}=0 \text {. }
$$

Without loss of generality it can be assumed that $\phi_{2}$ is non-zero. Eqs. (2.19), ..., (2.30) together with the conditions (1.1) and (4.1) yield

$$
\psi_{0}=\psi_{1}=\psi_{2}=\psi_{3}=0 \text {. }
$$

The Weyl tensor is therefore null. This completes the proof of the Theorem stated in the Introduction (the fact that the Weyl tensor must be non-zero is apparent from the subsequent calculations).

Stephani [4] has shown that, if the Einstein-Maxwell field is of embedding class one, the vector $l^{\alpha}$ is geodesic, hypersurface orthogonal and expansion free. Space-times admitting a congruence $l^{\alpha}$ having these properties have been discussed by KUNDT [8]. In particular the metric is of the form

$$
d s^{2}=-|d z-4 v s d u /(z+\bar{z})|^{2}-2 d u d v-H d u^{2}, s=+1 \text { or } 0 .
$$

The vectors $l^{\alpha}, n^{\alpha}, m^{\alpha}$ defined by

$$
l^{\alpha}=\delta_{2}^{\alpha}, n^{\alpha}=-\delta_{1}^{\alpha}+1 / 2 H \delta_{2}^{\alpha}-4 s v(z+\bar{z})^{-1}\left(\delta_{0}^{\alpha}+\delta_{0}^{\alpha}\right), m^{\alpha}=\sqrt{2} \delta_{0}^{\alpha}
$$

form a null tetrad. Here $\left(x^{1}, x^{2}, x^{0}, x^{\overline{0}}\right) \equiv(u, v, z, \bar{z})$.

Substituting the coordinates into the commutators yields

$$
\begin{aligned}
& x=\varrho=\sigma=\varepsilon=0, \\
& \alpha=\beta=1 / 2 \tau=1 / 2 \pi=-1 / 2 s \sqrt{2}(z+\bar{z})^{-1}, \\
& \lambda=\mu=4 s v(z+\bar{z})^{-2}, \\
& \nu=-1 / 2 \sqrt{2} \partial H / \partial \bar{z} \text { and } \gamma=-1 / 4 \partial H / \partial v .
\end{aligned}
$$

Substituting these values for the spin coefficients into the NewmanPenrose field equations gives

$$
\begin{gathered}
\Lambda=\phi_{0}=\phi_{1}=\psi_{0}=\psi_{1}=\psi_{2}=\psi_{3}=0 \\
\psi_{4}=-\partial^{2} H / \partial \bar{z}^{2}-2 H s(z+\bar{z})^{-2}-106 s v^{2}(z+\bar{z})^{-4} \\
+2 s v(z+\bar{z})^{-2} \partial H / \partial v+2 s(z+\bar{z})^{-1} \partial H / \partial \bar{z} \\
\phi_{22}=-\partial^{2} H / \partial z \partial \bar{z}-2 H s(z+\bar{z})^{-2}-106 s v^{2}(z+\bar{z})^{-4} \\
+2 s v(z+\bar{z})^{-2} \partial H / \partial v+s(z+\bar{z})^{-1}(\partial H / \partial z+\partial H / \partial \bar{z})
\end{gathered}
$$

with

$$
\partial^{2} H / \partial v^{2}=-24 s(z+\bar{z})^{-2} \text { and } \partial^{2} H / \partial v \partial z=48 s v(z+\bar{z})^{-3} .
$$

The Bianchi identities are identically satisfied and Maxwell's equations yield

$$
D \phi_{2}=\delta \phi_{2}=0 \text {. }
$$

The forms of the metric and tetrad are invariant under the transformations

$$
u^{\prime}=f(u), \quad v^{\prime}=v \dot{f}^{-1}(u) ; \quad z^{\prime}=z, \quad H^{\prime}=H \dot{f}^{-2}+2 \ddot{f} \dot{f}^{-3} v
$$


with

$$
\begin{gathered}
l^{\alpha^{\prime}}=\dot{f} l^{\alpha}, \quad n^{\alpha^{\prime}}=\dot{f}^{-1} n^{\alpha}, \quad m^{\alpha^{\prime}}=m^{\alpha} \\
v^{\prime}=v+f(u)(z+\bar{z})^{2 s}, \quad u^{\prime}=u, \quad z^{\prime}=z \\
H^{\prime}=H-16 s f^{2}(z+\bar{z})^{4 s-2}-32 v s f(z+\bar{z})^{2 s-2}-2 \dot{f}(z+\bar{z})^{2 s}
\end{gathered}
$$

with

$$
\begin{aligned}
l^{\alpha^{\prime}}= & l^{\alpha}, \quad n^{\alpha^{\prime}}=n^{\alpha}+8 s f^{2}(z+\bar{z})^{4 s-2} l^{\alpha} \\
& -2 \sqrt{2} s f(z+\bar{z})^{2 s-1}\left(m^{\alpha}+\bar{m}^{\alpha}\right), \quad m^{\alpha^{\prime}}=m^{\alpha} \\
& -2 \sqrt{2} s f(z+\bar{z})^{2 s-1} l^{\alpha},
\end{aligned}
$$

and, for $s=0$,

$$
\begin{gathered}
z^{\prime}=z+f(u), v^{\prime}=v-1 / 2(\dot{f} \bar{z}+\dot{\bar{f}} z), u^{\prime}=u, H^{\prime}=H+\ddot{f} \bar{z}+\ddot{\bar{f}} z-\dot{f} \dot{f} \\
l^{\alpha^{\prime}}=l^{\alpha}, \quad n^{\alpha^{\prime}}=n^{\alpha}+1 / 2 \dot{f} \dot{f} l^{\alpha} \\
+1 / 2 \sqrt{2} \dot{f} m^{\alpha}+1 / 2 \sqrt{2} \dot{\bar{f}} \bar{m}^{\alpha}, \quad m^{\alpha^{\prime}}=m^{\alpha}+1 / 2 \sqrt{2} \dot{f} l^{\alpha} .
\end{gathered}
$$

To complete the present work it is necessary to obtain those functions $H$ for which the metric (4.2) is of embedding class one. This is achieved by analysing the Gauss-Codazzi equations. The case of embeddings with $a_{33}=0$ is fairly straight forward and the results are summarized in section 5 (for $s=0$ ) and section 6 (for $s=1$ ). The case of embeddings with $a_{33} \neq 0$ is far more difficult and the appropriate functions $H$, although found in principle, are not displayed in a closed form. For this reason the explicit calculations are given in section 7 .

\section{Embeddings of the Space-Time $s=0$ with $a_{33}=0$}

With $a_{33}=s=0$ the Gauss-Codazzi equations admit a solution if and only if the function $H$ can be put in the form

$$
H=(\bar{A} z-A \bar{z})^{2},
$$

where $A$ is a function of $u$. One solution of the Gauss-Codazzi equations is then

$$
\begin{gathered}
e=+1 \\
a_{23}=-i \sqrt{2} \bar{A} \text { and } a_{22}=i(\dot{\bar{A}} z-A \bar{z})
\end{gathered}
$$

with all other components zero. The Eqs. (2.3) and (2.4) can be solved to give

$y^{Q}=C_{1}^{Q} v+C_{2}^{Q} u+C_{3}^{Q}+1 / 2 \sqrt{2}\left[R^{Q}(u)(A \bar{z}+\bar{A} z)-i S^{Q}(u)(A \bar{z}-\bar{A} z)\right]$ where $C_{1}^{Q}, C_{2}^{Q}, C_{3}^{Q}$ are constants and $R^{Q}, S^{Q}$ are real functions of $u$ satisfying the equations

$$
i S[A \dot{\bar{A}}-\bar{A} A]=-2 A \bar{A} \dot{R}-R[\dot{A} \bar{A}+\dot{\bar{A}} A]
$$


and

$\bar{A}[\ddot{\bar{A}}(R+i S)+\dot{\bar{A}}(\dot{R}+i \dot{S})+\bar{A}(\ddot{R}+i \ddot{S})]=\dot{\bar{A}}^{2}(R+i S)+4 \bar{A}^{2} A i S$.

As an explicit example of the embedding consider the case $A=+1$. Then the space-time

$$
d s^{2}=-d z d \bar{z}-2 d u d v-(z-\bar{z})^{2} d u^{2}
$$

is obtained from the flat space

$$
d s^{2}=-\left(d z^{1}\right)^{2}+\left(d z^{2}\right)^{2}-\left(d z^{3}\right)^{2}-\left(d z^{4}\right)^{2}+\left(d z^{5}\right)^{2}
$$

by the transformation

$$
\begin{aligned}
& z^{1}=1 / 2 i(z-\bar{z}) \cosh 2 u, \quad z^{2}=1 / 2 i(z-\bar{z}) \sinh 2 u \\
& z^{3}=1 / 2(z+\bar{z}), \quad z^{4}=1 / \sqrt{2}(u+v), \quad z^{5}=1 / \sqrt{2}(u-v) .
\end{aligned}
$$

\section{Embeddings of the Space-Time $s=1$ with $a_{33}=0$}

With $a_{33}=0, s=1$ the Gauss-Codazzi equations admit a solution if and only if the function $H$ can be put in the form

$$
H=-12 v^{2}(z+\bar{z})^{-2}+A+1 / 2 i B\left(z^{2}-\bar{z}^{2}\right)+C(z+\bar{z}),
$$

where $A, B$ and $C$ are real functions of $u$ with $A<0$. In this case

and

$$
\begin{gathered}
e=+1, \\
a_{23}=\sqrt{2} i A^{1 / 2}(z+\bar{z})^{-1}, \quad a_{22}=1 / 2 i A^{-1 / 2} A
\end{gathered}
$$

$$
y^{Q}=v M^{Q}(z+\bar{z})^{-1}-1 / 4 \dot{M}^{Q}(z+\bar{z})+i R^{Q}(z-\bar{z})+P^{Q}
$$

where $M^{Q}, R^{Q}$ and $P^{Q}$ are real functions of $u$ satisfying the equations

and

$$
\begin{aligned}
\ddot{M}^{Q} & =-4 B R^{Q} \\
\dot{R}^{Q} & =1 / 4 B M^{Q}
\end{aligned}
$$

$$
\dot{P}^{Q}=1 / 2 C M^{Q}+i A^{1 / 2} C_{1}^{Q}
$$

here $C_{1}^{Q}$ is a real constant.

As an explicit example of the embedding consider the case $A=-1$, $B=C=0$. Then the space-time

$$
\begin{aligned}
d s^{2}= & -\left|d z-4 v(z+\bar{z})^{-1} d u\right|^{2}-2 d u d v \\
& +d u^{2}+12 v^{2}(z+\bar{z})^{-2} d u^{2}
\end{aligned}
$$

is obtained from the flat space

$$
d s^{2}=-\left(d z^{1}\right)^{2}-\left(d z^{2}\right)^{2}+\left(d z^{3}\right)^{2}+\left(d z^{4}\right)^{2}-\left(d z^{5}\right)^{2}
$$


by applying the transformation

$$
\begin{gathered}
z^{1}=1 / 2 i(z-\bar{z}), \quad z^{2}=2 u v(z+\bar{z})^{-1}-1 / 2(z+\bar{z}), \quad z^{3}=u, \\
z^{4}=v\left(u^{2}+1\right)(z+\bar{z})^{-1}-1 / 2 u(z+\bar{z}), \quad z^{5}=v\left(u^{2}-1\right)(z+\bar{z})^{-1}-1 / 2 u(z+\bar{z}) .
\end{gathered}
$$

\section{Embeddings with $a_{33}=0$}

In this case the Codazzi equations yield $\tau=0$. Therefore only those space-times (4.2) with $s=0$ need be considered. For these space-times the Gauss equations $(2.7), \ldots,(2.18)$ give

with

$$
a_{11}=a_{12}=a_{13}=0, \quad a_{23}=A e^{i \theta}, \quad a_{33}=a_{34} e^{2 i \theta}
$$

and

$$
A^{2}-a_{22} a_{34}=-e \partial^{2} H / \partial z \partial \bar{z}
$$

Using (7.1) the non-trivial Codazzi equations can be written as

and

$$
\begin{aligned}
\theta_{; 3} A-\theta_{; 2} a_{34} e^{i \theta} & =0, \\
A_{; 3}-a_{34 ; 2} e^{i \theta}-i \theta_{; 2} a_{34} e^{i \theta} & =0, \\
a_{34 ; 4} e^{i \theta}-a_{34 ; 3} e^{-i \theta}+2 i \theta_{; 4} e^{i \theta} a_{34} & =0,
\end{aligned}
$$

$$
a_{22 ; 3}-a_{23 ; 2}-a_{33} 1 / 2 \sqrt{2} \partial H / \partial \bar{z}-a_{24} 1 / 2 \sqrt{2} \partial H / \partial z=0,
$$

where $a_{34}, A$ and $a_{22}$ are now independent of $v$.

If $\theta_{; 3}=0 \mathrm{Eq}$. (7.4) yields $\theta_{; 2}=0$ and so $\theta$ is a constant which can be made zero by means of the transformation $z^{\prime}=e^{i \theta} z$. Eq. (7.3) then gives, using the transformation (4.5),

where $x=z+\bar{z}$.

$$
H=H(x, u),
$$

Equations (7.5) and (7.6) yield

$$
A=\partial G / \partial u \text { and } a_{34}=-\sqrt{2} \partial G / \partial x
$$

where $G=G(x, u)$.

Only Eq. (7.7) remains to be satisfied. This equation can be written

$$
\begin{aligned}
\left(\frac{\partial G}{\partial x}\right)^{2} \frac{\partial^{2} G}{\partial u^{2}}+\left(\frac{\partial G}{\partial u}\right)^{2} & \frac{\partial^{2} G}{\partial x^{2}}-2 \frac{\partial G}{\partial u} \frac{\partial G}{\partial x} \frac{\partial^{2} G}{\partial u \partial x} \\
& +e \frac{\partial^{2} G}{\partial x^{2}} \frac{\partial^{2} H}{\partial x^{2}}-e \frac{\partial G}{\partial x} \frac{\partial^{3} H}{\partial x^{3}}+2\left(\frac{\partial G}{\partial x}\right)^{3} \frac{\partial H}{\partial x}=0 .
\end{aligned}
$$

Space-times given by (4.2) and (7.8) are therefore of embedding class one if Eq. (7.9) admits a solution for $G$ in terms of $H$. As an example consider the case $\partial H / \partial u=0$. Then one solution of (7.9) is

$$
e=+1, \sqrt{2} G=\log (\partial H / \partial x) \text {. }
$$


If $\theta_{; 3} \neq 0 \mathrm{Eq} .(7.4)$ gives

where

$$
A=P a_{34}
$$

$$
P=\left[e^{i \theta} \theta_{; 2} / \theta_{; 3}\right] \text {. }
$$

Eq. (7.6) is identically satisfied while Eqs. (7.5) and (7.7) can be written in terms of $a_{34}$ as

and

$$
P a_{34 ; 3}+P_{; 3} a_{34}-a_{34 ; 2} e^{i \theta}-i \theta_{; 2} a_{34} e^{i \theta}=0
$$

$$
\begin{gathered}
-e a_{34 ; 3} \partial^{2} H / \partial z \partial \bar{z}+e \sqrt{2} a_{34} \partial^{3} H / \partial z^{2} \partial \bar{z}+a_{34}^{3}\left(P P_{; 3}-e^{i \theta} P_{; 2}\right) \\
-a_{34}^{3} 1 / 2 \sqrt{2}\left(e^{2 i \theta} \partial H / \partial \bar{z}+\partial H / \partial z\right)=0 .
\end{gathered}
$$

Eq. (7.11) is an equation for $a_{34 ; 3}$ whilst (7.10) can be rewritten as an equation for $a_{34 ; 2}$. The integrability condition for these equations will give a polynomial in $a_{34}$. If this is solved and the resulting expression for $a_{34}$ is substituted back into (7.10) and (7.11) then two conditions on $H$ are obtained which, together with

$$
\left(\partial^{2} H / \partial z^{2}\right)\left(\partial^{2} H / \partial \bar{z}^{2}\right)=\left(\partial^{2} H / \partial z \partial \bar{z}\right)^{2}
$$

[see Eq. (7.3)], will form a set of sufficient conditions for embedding class one. This then exhausts all possible Einstein-Maxwell fields of embedding class one.

The author would like to thank Professor F.A. E. PrranI for his helpful comments on the manuscript.

\section{References}

1. Collinson, C. D.: to be published in J. Math. Phys.

2. Thomas, T. Y.: Acta. Math. 67, 169 (1936).

3. Szekeres, P.: Nuovo Cimento XL 111 A, 1062 (1966).

4. Stephani, H.: Commun. math. Phys. 4, 137 (1967).

5. Newman, E., and R. Penrose: J. Math. Phys. 3, 566 (1962).

6. -, - J. Math. Phys. 4, 998 (1963).

7. Eisenhart, L. P.: Riemannian Geometry, p. 97. Princeton. New Jersey: Princeton University Press 1925.

8. Kundt, W.: Z. Physik 163, 77 (1961).

Dr. C. D. Collinson

Department of Applied Mathematics

University of Hull

Hull, Great Britain 